\title{
A novel telerehabilitation with educational program for caregivers using telelecture is a feasible procedure for fall prevention in elderly people - A pilot study
}

\section{Kentaro Moriichi}

Asahikawa Medical University

Mikihiro Fujiya ( $\square$ fjym@asahikawa-med.ac.jp )

Asahikawa Medical University

Takanori Ro

Asahikawa Medical University

Tetsuo Ota

Asahikawa Medical University

Hitomi Nishimiya

Asahikawa Medical University

Mariko Kodama

Asahikawa Medical University

Nana Yoshida

Asahikawa Medical Universtity

Yukari Hattori

Asahikawa Medical University

Tetsuya Hosokawa

Asahikawa Medical University

Hohei Hishiyama

Chuwa Clinic

Masao Kunimoto

Kunimoto Hospital

Hiroki Hayashi

Asahikawa Medical University

Hiroyuki Hirokawa

Asahikawa Medical University

Akitoshi Yoshida

Asahikawa Medical University 
Research article

Keywords: Falls, Rehabilitation, Physical Activity, Balance, Telemedicine

Posted Date: August 21st, 2020

DOI: https://doi.org/10.21203/rs.3.rs-49205/v1

License: (c) (i) This work is licensed under a Creative Commons Attribution 4.0 International License. Read Full License 


\section{Abstract}

Background: The importance of fall prevention rehabilitations has been well recognized. Recently, telerehabilitation was developed, however, there have been no reports on the use of telerehabilitation with direct support from specialists for fall prevention among the elderly. We herein reported telerehabilitation by carers educated by our novel educational program.

Methods: Nine elderly people in two nursing homes were enrolled using our original criteria. Carers are educated with our educational program using telelecture system. Telerehabilitation was performed by carers following the instruction from rehabilitation specialists in Asahikawa Medical University using the telemedicine system every 2-4 weeks for three months. Carers were assessed with our original questionnaire before and after the telelecture. Berg Balance Scale (BBS), Timed Up \& Go test (TUG test), Hand-held dynamometer (HHD) and Mini-Mental State Examination (MMSE) were assessed before and after telerehabilitation.

Results: The average number of times to perform telerehabilitation in all institutes was 4.7. Levels of understanding of carers were significantly increased after the telelecture. No adverse event occurred during the study period. Median BBSs before and three months after telerehabilitation were 43 and 49, respectively. Those of TUG test, right and left HHD and MMSE were 17.89 and 18.53, 7.95 and 11.55, 9.85 and 13.20 , and 16 and 19 , respectively. All results were improved after telerehabilitation.

Conclusions: Our telerehabilitation program exhibited significant effects in elderly people as well as levels of understanding rehabilitation of caregivers in the facilities for the elderly people safely.

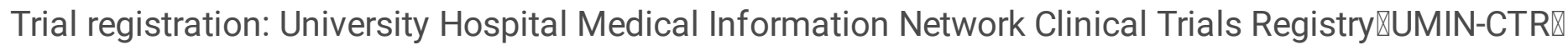
UMIN000041439, August $17^{\text {th }}, 2020$

\section{Background}

It is estimated that approximately $30 \%$ of community dwelling people of $\geq 65$ years of age experience a fall at least once per year [1]. Residents of institutions showed a higher rate of fall in comparison to elderly people living in houses [2]. In Japan, Yasumura et al. reported that the prevalence of falls was 12.3-26.9\% [3]. Falls, as well as pneumonia, are events that can threaten the health status of elderly adults, and which have the potential to adversely impact their activities of daily living. Indeed, falls account for $19.3 \%$ of deaths in Japan [3]. It is therefore crucial to prevent falls among the elderly and rehabilitation for fall prevention should be widely performed because few elderly people recover their muscle strength and body balance without rehabilitation.

Although various rehabilitation strategies have been applied in the clinical setting for a long time, most require face-to-face therapy. Thus, the patients who want to receive rehabilitation have to travel to rehabilitation centers or hospitals, which involves time and financial costs [4]. In addition, nursing staff in rural areas cannot easily obtain the latest knowledge on rehabilitation because it can be difficult to attend 
medical lectures or meetings [5]. With regard to rehabilitation, medical inequality between rural and urban populations have been an object of discussion for many years [6-9]. Wakerman and Humphreys reported the health disadvantage of rural residents in Australia and noted that they experienced poorer health outcomes in comparison to urban residents [10]. In line with these findings, Bradford et al. showed that the mortality and life expectancy of people living in rural areas were poorer in comparison to people living in urban areas [11].

Recently new telecommunication-based methods that enable the patients to receive rehabilitation in their own home have been developed and applied in the field of rehabilitation [12]. While telerehabilitation has been clinically applied, most patients have been middle-aged adults with diseases including multiple sclerosis [13], stroke [14] and cardiac disease [15]. Thus, the efficacy of telerehabilitation for fallprevention in elderly people has been totally unclear. In this pilot study, we proposed a novel procedure for fall-preventive telerehabilitation for elderly people, which combined a physical therapist (PT)-assisted and personalized telerehabilitation program with a caregiver-education program.

\section{Methods}

\section{Telemedicine systems}

A telemedicine system was installed in the Telemedicine Center of Asahikawa Medical University (Figure 1A). The VidyoRoom HD230 software program (Vidyo, Inc., Hackensack, USA) was used for telemedicine conferences, lectures and rehabilitation sessions in the telemedicine center. The VidyoMobile software program (Vidyo, Inc., Hackensack, USA) was used in the institutions, which included elderly group homes and nursing homes. Each institution can connect to the telemedicine network via a broadband Internet connection (e.g., an asymmetric digital subscriber line [ADSL] or fiberoptic line) using a tablet or laptop PC. The maximum image quality of the system is $1080 \mathrm{pHD}$ and the images are shrinked by H.264/Scalable Video Coding (SVC), an original signaling protocol developed by Vidyo, Inc.. The security of our telemedicine system consists of Advanced Encryption Standard (AES) encryption and a Secure Sockets Layer (SSL) (Figure 1B). Two tablet terminals are used as video cameras in each institution. To view all participants, one tablet terminal was placed diagonally to the participant and the other was placed by their side (Figure 1C). Specialists, including medical doctors (MDs) from the rehabilitation department, PTs and nurses gave lectures to staff members in all institutions and created a personalized telerehabilitation plan for each patient.

\section{Education for caregivers}

Before the telerehabilitation was started, medical staff (MDs, nurses and PTs) in Asahikawa Medical University gave lectures on rehabilitation to staff members at elderly institutions, including caregivers and nurses using the teleconference system. In these lectures, nurses talked about the mechanism of falling and fall prevention and PTs explained the actual methods of the rehabilitation using original materials based on the guidelines or consensus. To evaluate the efficacy of the lectures, 13 staff members from elderly institutions took the same test regarding knowledge in relation to falls before and after the 
lectures. The test contained 20 questions in eight categories: the relationship between falls and Living environments, bone fracture, medication use, a past history of falls, disease, the reasons for requiring nursing care, and dementia (Figure 2).

\section{Participant selection}

\section{Inclusion criteria}

The inclusion criteria were as follows: 1) clear consciousness ; 2) stable respiratory and circulation status ;3) ability to communicate and exercise while following directions; 4) ability to walk with or without walk aids; 5) ability to exercise with a caregiver's assistance with or without knowledge about rehabilitation, 6) the provision of informed consent by the participant or their family. Even when a participant met all of these conditions above, rehabilitation was cancelled when the following conditions were not met just before the rehabilitation session: 1 ) a resting heart rate of $40-120$ beats $/ \mathrm{min} ; 2$ ) a resting systolic blood pressure from $70-200 \mathrm{mmHg} ; 3$ ) a resting diastolic blood pressure of $<120 \mathrm{mmHg}$; 4) no effort angina pectoris; 5) no atrial fibrillation with remarkable bradycardia or tachycardia; 6) a stable circulation status without recent history of cardiac infarction; 7) no remarkable arrhythmia; 8) no chest pain at rest; 9) no palpitations, shortness of breath or chest pain; 10) no nystagmus, cold sweat or nausea; 11) body temperature $\left.<38^{\circ} \mathrm{C} ; 12\right)$ blood oxygen saturation $\left(\mathrm{SaO}_{2}\right)$ at rest $>90 \%$.

\section{Exclusion criteria}

The following exclusion criteria were applied: 1) resting systolic blood pressure outside the range of 70$200 \mathrm{mmHg}$; 2) resting diastolic blood pressure $\geq 120 \mathrm{mmHg}$; 3 ) resting heart rate of $\leq 40 \mathrm{beats} / \mathrm{min}$ or $\geq 120 \mathrm{~min}$; 4) poorly controlled arrhythmia; 5 ) ischemic heart disease such as acute myocardial infarction, unstable angina pectoris with symptoms of heart failure; 6) pulmonary disease with cyanosis and/or a $\mathrm{SaO}_{2}$ value of $\leq 90 \%$; 7) poorly controlled diabetes mellitus (DM); DM with complications, including neuropathy, retinopathy and nephropathy.

\section{Questionnaire}

We modified the Physical Activity Readiness Questionnaire (PAR-Q) to assess the health status of the telerehabilitation participants. The participants or their family members answered the questions. The questionnaire consists of ten questions related to the patient's health history, current symptoms, and risk factors to determine the safety of rehabilitation and possible risks associated with their participation (Figure 3A).

\section{Fall screening sheet}

To evaluate the indications for rehabilitation, the participants' information was searched by nursing staff using the fall screening sheet. The fall screening sheet contained information about the participants, including their age, present illness, past history of falls and stroke, nursing care levels, usage of fall prevention alarm systems, and rehabilitation experience (Figure 3B) 


\section{Selection of participants}

Based on the above information, MDs, PTs and nurses in Asahikawa Medical University discussed the selection of participants with staff members of elderly institutions using telemedicine systems, then the telerehabilitation participants were selected.

\section{Telerehabilitation programs}

MDs, nurses and PTs selected a personalized program for each participant from the following rehabilitation menus based on the "Questionnaire" and "Fall screening sheet" as well as the physical and mental condition of each participant using the telemedicine system. The rehabilitation menus contained six exercises (rising training, high knee training, straight leg raise training, side lying leg lift training, heel raise training and a knee straightening exercise), which mainly focused on muscular strength training of the lower limbs and the improvement of sitting balance. At the first session, the rehabilitation session was observed by MDs, nurses and PTs using the telerehabilitation system. The participants were required to continue the rehabilitation two or three times per week by themselves with caregiver's support in their nursing home. The interval between rehabilitation sessions depended on the participants' rehabilitation program and condition. All participants underwent telerehabilitation five times in three months (first time, one week, one month, two months and three months later, respectively). (Figure 3C-E)

\section{Points of evaluation}

The efficacy of the telerehabilitation for each participant was evaluated using quantitative scales, including muscle strength, the Berg Balance Scale (BBS) [16], the Timed Up \& Go test (TUG test) [17] and the Mini-Mental State Examination (MMSE) [18].

\section{Muscle strength}

We measured knee extensor muscle strength using hand-held dynamometer (HHD; $\mu$ Tas F-1, Anima Corp., Tokyo) [19]. Briefly, with the participant sitting on a chair, the HHD sensor is attached to the limb with a belt that is anchored to a fixed structure. The participant extends the limb, and then knee extensor muscle strength is measured using the HHD.

\section{Berg Balance Scale}

To evaluate participants' balance ability, all participants were assessed by the BBS [16]. The BBS is thought to be reliable and widely accepted method for the evaluation of balance [20]. Briefly, this scale contains 14 items related to sitting balance, standing balance and dynamic balance. The scores for each item range from 0 to 4 , with a score of 0 representing inability to complete the task and a score of 4 representing independent completion of the task.

\section{Timed Up \& Go test}


The TUG test has been widely used to evaluate basic mobility maneuvers that are frequently performed by the elderly population $[17,21]$. The TUG test measures the time that it takes for a person to rise from a chair, walk three meters, turn around, walk back to the chair, and sit down [17].

\section{Mini-Mental State Examination (MMSE)}

The MMSE was developed to measure cognitive impairment in 1975 [18] and is currently used for dementia screening. The MMSE is a 30-point questionnaire, with five sections (orientation [10 points], registration [3 points], attention and calculation [5 points], recall [3 points] and language [9 points]) [22]. The cut-off score for the diagnosis of dementia is reported to be $<24$, which is considered to be a standard criterion $[23,24]$.

\section{Results}

\section{Participants}

Nine elderly people were selected from 24 people receiving care in elderly institutions using the abovedescribed selection form. All participants were living in nursing homes. The mean age of the participants was $84.6 \pm 4.5$ years. One participant was male; 8 were female. Of the nine participants, two participants used walking aids and four participants had past history of fall on at least one occasion (Table). Written informed consent was obtained from all of the participants.

\section{Nursing staff education}

Nineteen staff members of elderly institutions including nurses and caregivers took the test. The average score before the lecture was 15.3, while that after the lecture was 18.3. The lecture significantly improved the total test score $(p<0.001)$. The category that showed the lowest score before the lecture was the reasons for requiring nursing care", which showed significant improvement after the lecture, illustrating the efficacy of the telerehabilitation education program for staff members of elderly institutions (Figure 4).

\section{Berg Balance Scale}

Four of the nine participants showed improved BBS values after telerehabilitation. The average BBS values before rehabilitation and after one, two, and three months of rehabilitation were 44.6, 45.2, 46.0 and 46.3 , respectively, and increased with time (Figure 5A). This suggests that the telerehabilitation is a feasible option for improving the body balance of elderly people.

\section{Timed Up \& Go test}

Eight participants were assessed by TUG test while one participant declined to participate in the first TUG test due to anoesia. Four of the 8 remaining participants showed improvement after 3 months of rehabilitation. However, the average of TUG scores of the participants showed no significant change from 
pre-rehabilitation to post-rehabilitation, indicating that the telerehabilitation may improve the exercise ability for some elderly people (Figure 5B).

\section{Muscle strength}

Five of the nine participants showed improved left knee muscle strength. After rehabilitation, the median right and left knee extensor strength increased from $7.95 \mathrm{~kg}$ and $9.85 \mathrm{~kg}$ to $11.55 \mathrm{~kg}$ and $13.20 \mathrm{~kg}$, respectively, suggesting that the telerehabilitation improved the muscle strength of the lower limbs (Figure 5C).

\section{Mini-Mental State Examination (MMSE)}

The median MMSE scores before and after 3 months of rehabilitation were 19.0 and 16.0, respectively. Despite the short study period, five of nine participants showed improvement (Figure 5D), suggesting the usefulness of telerehabilitation for improving the cognitive status as well as physical activity.

\section{Adverse events}

All participants completed all procedures without any adverse events. No participants fell or showed any health problems during telerehabilitation and home exercise, suggesting the safety of the telerehabilitation program.

\section{Discussion}

This pilot study demonstrated the efficacy of the telerehabilitation for preventing falls in the elderly population. All participants could complete the telerehabilitation program, including the exercises, by themselves with support from caregivers. Around half of participants showed improved BBS or TUG values. It is noteworthy that muscle strength, particularly right knee extension muscle strength, improved in all participants. These results indicated that telerehabilitation is feasible for the prevention of falls in the elderly population.

Several studies have shown the efficacy of fall prevention rehabilitation for nursing home residents [25, 26]. It is crucial to prevent falls in the elderly population as they are thought to be the main cause of fatal and non-fatal injuries in elderly individuals. It is well-known that the risk of falls increases with age [27]. Recent meta analyses and systematic reviews revealed the efficacy of fall prevention intervention in community-dwelling older people [28-31]. Community-based fall prevention interventions are low cost and low-tech programs, which can achieve a $25-30 \%$ reduction in falls per year after the program [32]. Thus, the efficacy of conventional fall prevention intervention has been well recognized. However, interventions are often provided by non-medical specialists or less-integrated staff [32] and face-to-face procedures are required, which would make these interventions difficult to implement in rural areas. To solve this problem, it is crucial to establish standardized telerehabilitation methods. While telecommunicationbased rehabilitation methods have recently been developed [12], the methods have mostly been applied for the rehabilitation of middle-aged patients, with diseases including multiple sclerosis [13], stroke [14] 
and cardiac disease [15], rather than elderly people. There have been no trials of telerehabilitation focused on preventive rehabilitation for general elderly people. In addition, at present, the quality of the rehabilitation is not secured [32]. The present study suggests that our telerehabilitation system is a feasible option for the rehabilitation of elderly people in rural areas.

Regarding the efficacy and safety of rehabilitation, low levels of knowledge in relation to rehabilitation are another problem regarding the rehabilitation of elderly people. Our telerehabilitation system therefore included an education program. The fall-associated knowledge of staff members of elderly institutions was significantly increased after lectures by medical specialists using a teleconference system. The medical specialists, including MDs, nurses and PTs as well as staff members of elderly institutions (including caregivers) did not need to go anywhere, which reduced the time and costs, particularly for people in rural areas.

When conducting telerehabilitation, it is extremely important to secure the safety of the participant. For this reason, we set strict inclusion criteria for this telerehabilitation program and measured the participants' vital signs and asked participants about their condition via the telemedicine system before and after the telerehabilitation sessions. In addition, during telerehabilitation and home exercise, nursing staff members who had received a lecture on rehabilitation for fall prevention observed the participants on an individual basis. As a result, no adverse events were observed during the present study.

From an economic point of view, Dieleman et al. estimated that spending on personal healthcare for falls in the United States amounted to $\$ 76.3$ billion [33]. Using the Medicare Current Beneficiary Survey data Florence et al. estimated that the annual cost of falls was $\$ 49.5$ billion dollars [34]. Telerehabilitation can be expected to reduce costs in relation to hospital visits and staff education, particularly in rural areas; thus, telerehabilitation has the potential to reduce medical costs. Regarding our selection criteria, 9 of 24 elderly people met the inclusion criteria and approximately half of the participants showed improvement in their physical activity. A further analysis of a larger population of elderly people will be useful for determining the proportion of elderly people for whom telerehabilitation is appropriate and estimating the associated savings in medical costs.

Improvement of the cognitive status is known to be positive for the quality of life and survival of elderly people. Previous reports have shown that physical exercises had a good influence on the cognitive status of elderly people, particularly those with mild cognitive impairment $[35,36]$. Although this study aimed to confirm the efficacy of the telerehabilitation for improving physical activity, our telerehabilitation program unexpectedly improved the MMSE scores as well as physical scales. It is still unclear why physical exercise improves the cognitive function; however, the results of the present study suggest that our telerehabilitation system may improve the cognitive function without face-to-face procedures. Our telerehabilitation system is expected to improve the cognitive function of elderly people living in urban as well as rural areas.

The present study was associated with some limitations. First, this study employed a single-arm design. If the muscle strength or body balance improved in a large number of people who did not receive 
telerehabilitation, then our data would show less impact. However, it is known that almost no elderly people can recover muscle strength and body balance without rehabilitation. While the present study did not employ a comparative design, it was still significant that the quantitative scales regarding muscle strength and body balance were improved in approximately half of the participants. Second, the duration of the study was only 3 months, which was considered to be too short to assess the incidence of falls. However, it is known that the risk of falls is closely associated with muscle strength and body balance. The present study clearly showed that our telerehabilitation could improve muscle strength and body balance, suggesting a preventive effect against falls in elderly people. Third, because only nine participants were enrolled in this study, the statistical power was limited. A further analysis with a larger study population is needed to reveal the efficacy and safety of telerehabilitation for fall prevention in the elderly population.

\section{Conclusion}

This study illustrated that our telerehabilitation program, which included a caregiver-education program using telelectures significantly improved the exercise ability of the elderly participants without adverse events, as well as the level of understanding regarding rehabilitation among caregivers in elderly institutions. Considering that nine participants were selected from twenty-four residents, falls prevention rehabilitation may be indicated for a large number of elderly people. We are conducting a randomized controlled study with a larger study population to validate the effectiveness of this telerehabilitation system. Telerehabilitation can be expected to play an important role in improving the physical status of elderly people, and to be beneficial for reducing the costs associated with rehabilitation.

\section{Abbreviations}

ADSL: asymmetric digital subscriber line

AES: Advanced Encryption Standard

BBS: Berg Balance Scale

DM: diabetes mellitus

HHD: Hand-held dynamometer

MD: medical doctor

MMSE: Mini-Mental State Examination

PAR-Q: Physical Activity Readiness Questionnaire

PT: physical therapist 
Sa02: blood oxygen saturation

SSL: Secure Sockets Layer

SVC: Scalable Video Coding

TUG test: Timed Up \& Go test

\section{Declarations}

\section{Ethics approval and consent to participate}

This study (UMIN000041439) was approved by the institutional review board of Asahikawa Medical University (IRB \# 17191). All participants provided written, informed consent.

\section{Consent for publication}

Consent for publication was obtained from all participants

\section{Availability of data and material}

The datasets used and analyzed during the current study are available from the corresponding author on reasonable request.

\section{Competing of Interest}

A.Y. received a research grant from Kanamic Network Co., LTD. MF, KM, HN, NY and TH belong to Joint Research Department collaborated with Kanamic Network Co., LTD.

\section{Funding}

This work was supported by Grant-in-Aid for Scientific Research(B) No. $19 \mathrm{H} 03973$ (A. Yoshida). This grant supported to purchase goods for telerehabilitation and telelecture, such as tablet terminals, video cameras, various sensors, vital sign and muscle strength measuring equipments, personal computer monitors and so on.

\section{Authors' contributions}

$\mathrm{KM}, \mathrm{MF}, \mathrm{TR}$ and $\mathrm{HN}$ contributed equally to this study and provided major input on the conceptual development of the studies, wrote the manuscript, and supervised all investigations. TH and HN supported the participants and nursing staff members and collected the data. TR and TO performed telerehabilitation and determined the inclusion criteria. TR, $\mathrm{HN}$ and MK gave lectures to nursing staff members. NY collected data regarding the health condition of the participants and obtained their informed consent. YH, H. Hayashi, H. Hishiyama, M. Kunimoto and H. Hirokawa helped to perform the 
study. AY supervised the study and reviewed the manuscript. All of the authors read and approved the final manuscript.

\section{Acknowledgements}

The authors wish to thank Mr. D. Mikami for their advice on telemedicine systems.

\section{References}

1. Gillespie LD, Robertson MC, Gillespie WJ, et al. Interventions for preventing falls in older people living in the community. Cochrane Database Syst Rev. 2012;12:CD007146.

2. Ayed I, Ghazel A, Jaume-I-Capó A, et al. Feasibility of Kinect-Based Games for Balance Rehabilitation: A Case Study. J Healthc Eng. 2018;2018:7574860.

3. Yasumura S, Hasegawa M. Incidence of Falls among the Elderly and Preventive Efforts in Japan. 2009;52:231-236.

4. Dávalos ME, French MT, Burdick AE, et al. Economic evaluation of telemedicine: review of the literature and research guidelines for benefit-cost analysis. Telemed J E Health. 2009;15:933-48.

5. Dlouhý M. Measuring Geographic Inequalities: Dealing with Multiple Health Resources by Data Envelopment Analysis. Front Public Health. 2018;6:53.

6. Pantyley V. Health inequalities among rural and urban population of Eastern Poland in the context of sustainable development. Ann Agric Environ Med. 2017;24:477-483.

7. Smith K.B., Humphreys J.S., Wilson M.G.. Addressing the health disadvantage of rural populations: how does epidemiological evidence inform rural health policies and research? J. Rural Health. 2008;16:56-66.

8. Eberhardt M.S., Pamuk E.R. The importance of place of residence: examining health in rural and nonrural areas. J. Public Health. 2004;94:1682-1686.

9. Ricci-Cabello I, Ruiz-Perez I, Rojas-García A, et al. Improving diabetes care in rural areas: a systematic review and meta-analysis of quality improvement interventions in OECD countries. PLOS ONE. 2013;8 Article e84464.

10. Wakerman J, Humphreys JS. Sustainable primary health care services in rural and remote areas: innovation and evidence. The Australian Journal of Rural Health. 2011;19:118-124.

11. Bradford NK, Caffery LJ, Smith AC. Telehealth services in rural and remote Australia: a systematic review of models of care and factors influencing success and sustainability. Rural Remote Health. 2016;16:4268.

12. Botsis T, Demiris G, Pedersen S, et al. Home telecare technologies for the elderly. J Telemed Telecare. 2008;14:333-7.

13. Amatya B, Khan F, Galea M. Rehabilitation for people with multiple sclerosis: an overview of Cochrane Reviews. Cochrane Database Syst Rev. 2019;1:CD012732. 
14. Tchero H, Tabue Teguo M, Lannuzel A, et al. Telerehabilitation for Stroke Survivors: Systematic Review and Meta-Analysis. J Med Internet Res. 2018;20:e10867.

15. Dinesen B, Nielsen G, Andreasen JJ, et al. Integration of Rehabilitation Activities Into Everyday Life Through Telerehabilitation: Qualitative Study of Cardiac Patients and Their Partners. J Med Internet Res. 2019;21:e13281.

16. Berg K, Wood-Dauphine S, Williams $\mathrm{Jl}$, et al. Measuring balance in the elderly: preliminary development of an instrument. Physiotherapy Canada. 1989;41:304-311.

17. Podsiadlo D, Richardson S. The timed 'Up \& Go': a test of basic functional mobility for frail elderly persons. Am. Geriatr. Soc. 1991;39:142-148.

18. Folstein MF, Folstein SE, McHugh PR. "Mini-mental state". A practical method for grading the cognitive state of patients for the clinician. J Psychiatr Res.1975;12:189-98.

19. Katoh M, Yamasaki H. Test-Retest Reliability of Isometric Leg Muscle Strength Measurements Made Using a Hand-Held Dynamometer Restrained by a Belt: Comparisons during and between Sessions. Phys. Ther. Sci. 2009;21:239-243.

20. Downs S, Marquez J, Chiarelli P. The Berg Balance Scale has high intra- and inter-rater reliability but absolute reliability varies across the scale: a systematic review. J Physiother. 2013;59:93-9.

21. Lee J, Geller Al, Strasser DC. Analytical review: focus on fall screening assessments. PM R. 2013;5:609-21.

22. Palsetia D, Rao GP, Tiwari SC, et al. The Clock Drawing Test versus Mini-mental Status Examination as a Screening Tool for Dementia: A Clinical Comparison. Indian J Psychol Med. 2018;40:1-10.

23. Dick JP, Guiloff RJ, Stewart A, et al. Mini-Mental State Examination in neurological patients. J Neurol Neurosurg Psychiatry. 1984;47:496-499.

24. Bour A, Rasquin S, Boreas A, et al. How predictive is the MMSE for cognitive performance after stroke? J Neurol. 2010;257:630-637.

25. Jensen J, Lundin-Olsson L, Nyberg L, et al. Fall and injury prevention in older people living in residential care facilities. A cluster randomized trial. Ann Intern Med. 2002;136:733-741.

26. Becker $\mathrm{C}$, Kron M, Lindemann $\mathrm{U}$, et al. Etfectiveness of a multifaceted intervention on falls in nursing home residents. J Am Geriat Sroc. 2003;51:306-31.

27. McCarthy M. Falls are leading cause of injury deaths among older people, US study finds. 2016;354:i5190.

28. Lamb SE, Jørstad-Stein EC, Hauer K, et al. Development of a common outcome data set for fall injury prevention trials: the Prevention of Falls Network Europe consensus. J Am Geriatr Soc. 2005;53:161822.

29. Sherrington C, Whitney JC, Lord SR, et al. Effective exercise for the prevention of falls: a systematic review and meta-analysis. J Am Geriatr Soc. 2008;56:2234-43.

30. Gates S, Fisher JD, Cooke MW, et al. Multifactorial assessment and targeted intervention for preventing falls and injuries among older people in community and emergency care settings: 
systematic review and meta-analysis. 2008;336:130.

31. Choi YS, Lawler E, Boenecke CA, et al. Developing a multi-systemic fall prevention model, incorporating the physical environment, the care process and technology: a systematic review. $J \mathrm{AdV}$ Nurs. 2011;67:2501-24.

32. Howland J, Hackman $\mathrm{H}$, Taylor A, et al. Older adult fall prevention practices among primary care providers at accountable care organizations: A pilot study. PLoS One. 2018;13:e0205279.

33. Dieleman JL, Baral R, Birger M, et al. Us spending on personal health care and public health, 19962013. 2016;316:2627-2646.

34. Florence CS, Bergen G, Atherly A, et al. The Medical Costs of Fall Injuries among Older Adults. J Am Geriatr Soc. 2018;66:693-698.

35. Northey JM, Cherbuin N, Pumpa KL, Smee DJ, Rattray B. Exercise interventions for cognitive function in adults older than 50: a systematic review with meta-analysis. Br J Sports Med. 2018;52:154-160.

36. Netz Y. Is There a Preferred Mode of Exercise for Cognition Enhancement in Older Age? - A Narrative Review. Front Med (Lausanne). 2019;6:57.

\section{Tables}

Table

\begin{tabular}{|c|c|c|c|c|c|}
\hline & age-ranges & Gender & $\begin{array}{c}\text { Walking } \\
\text { aids }\end{array}$ & $\begin{array}{l}\text { History } \\
\text { of falls }\end{array}$ & Present illness \\
\hline Case 1 & $70 \mathrm{~s}$ & $\mathrm{~F}$ & No & + & $\mathrm{DM}^{*}, \mathrm{HT}^{\dagger}$, Breast cancer $\mathrm{p} / \mathrm{o}$ \\
\hline Case 2 & $90 \mathrm{~s}$ & $\mathrm{~F}$ & No & + & Dementia, DL:, Gonitis, old Tbc \\
\hline Case 3 & $80 \mathrm{~s}$ & $\mathrm{~F}$ & Yes & - & Dementia, Collagen disease, Cataract \\
\hline Case 4 & $80 \mathrm{~s}$ & $\mathrm{~F}$ & No & - & Dementia, HT \\
\hline Case 5 & $70 \mathrm{~s}$ & $\mathrm{~F}$ & Yes & - & Dementia, $\mathrm{CKD}^{\S}$ \\
\hline Case 6 & $80 \mathrm{~s}$ & $\mathrm{~F}$ & No & - & Dementia \\
\hline Case 7 & $80 \mathrm{~s}$ & $\mathrm{~F}$ & No & + & Dementia, HT, femoral fracture \\
\hline Case 8 & $80 \mathrm{~s}$ & $\mathrm{~F}$ & No & - & Dementia, HT, Osteoporosis \\
\hline Case 9 & $80 \mathrm{~s}$ & $\mathrm{M}$ & No & - & Dementia \\
\hline
\end{tabular}

\section{Figures}


A

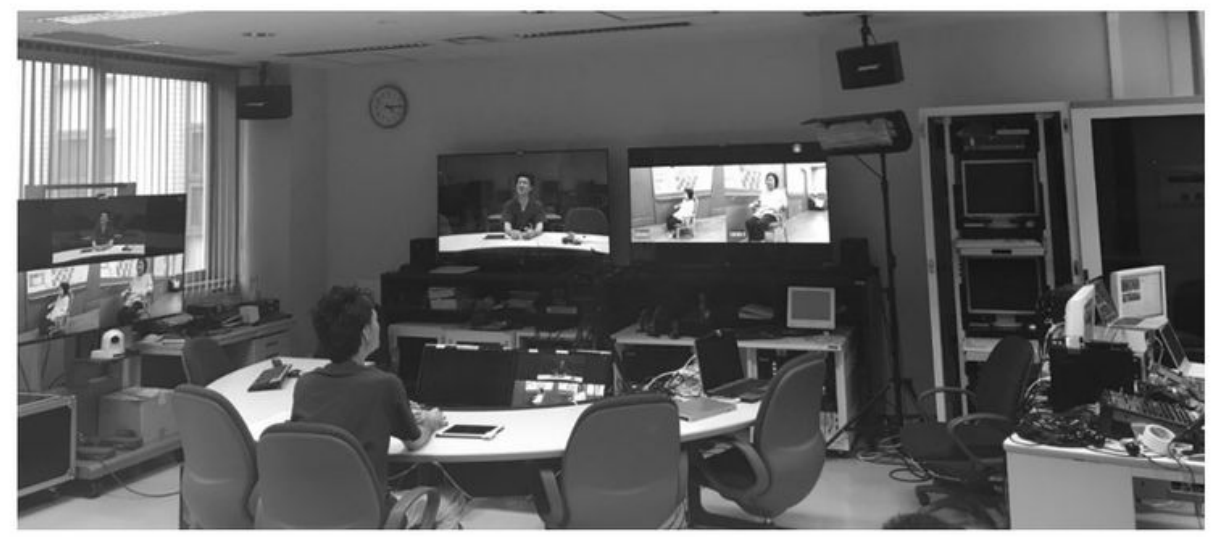

$\mathrm{B}$

Elderly institution

Tablet terminal

$\mathrm{C}$

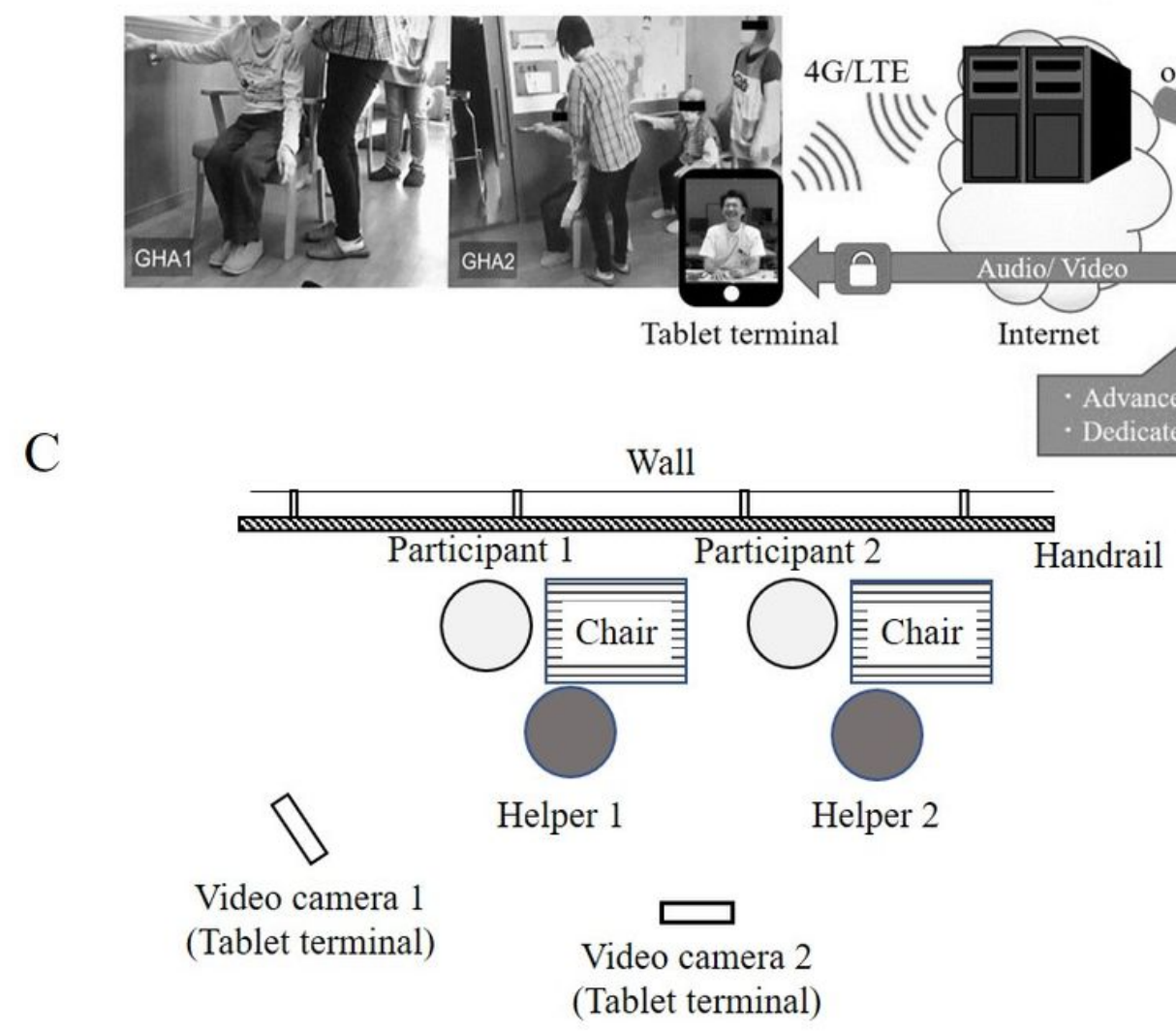

web conferencing server
Asahikawa Medical University Telemedicine center

\section{Figure 1}

The Telemedicine center and a Bird's-eye view of telerehabilitation A distant view of the Telemedicine Center in Asahikawa Medical University; the lecturer sits in front of the video camera and monitors. The operator monitors the telerehabilitation session (A). A schematic illustration of the telemedicine system. Each institution can connect to the telemedicine network via a broadband Internet connection (e.g., asymmetric digital subscriber line [ADSL] or fiberoptic connection using a tablet or laptop PC (B).

Participants exercise along the wall with a handrail to prevent staggering and caregivers stand beside the participants. One video camera (tablet terminal) is placed diagonally to the participant; the other is placed by their side (C). 


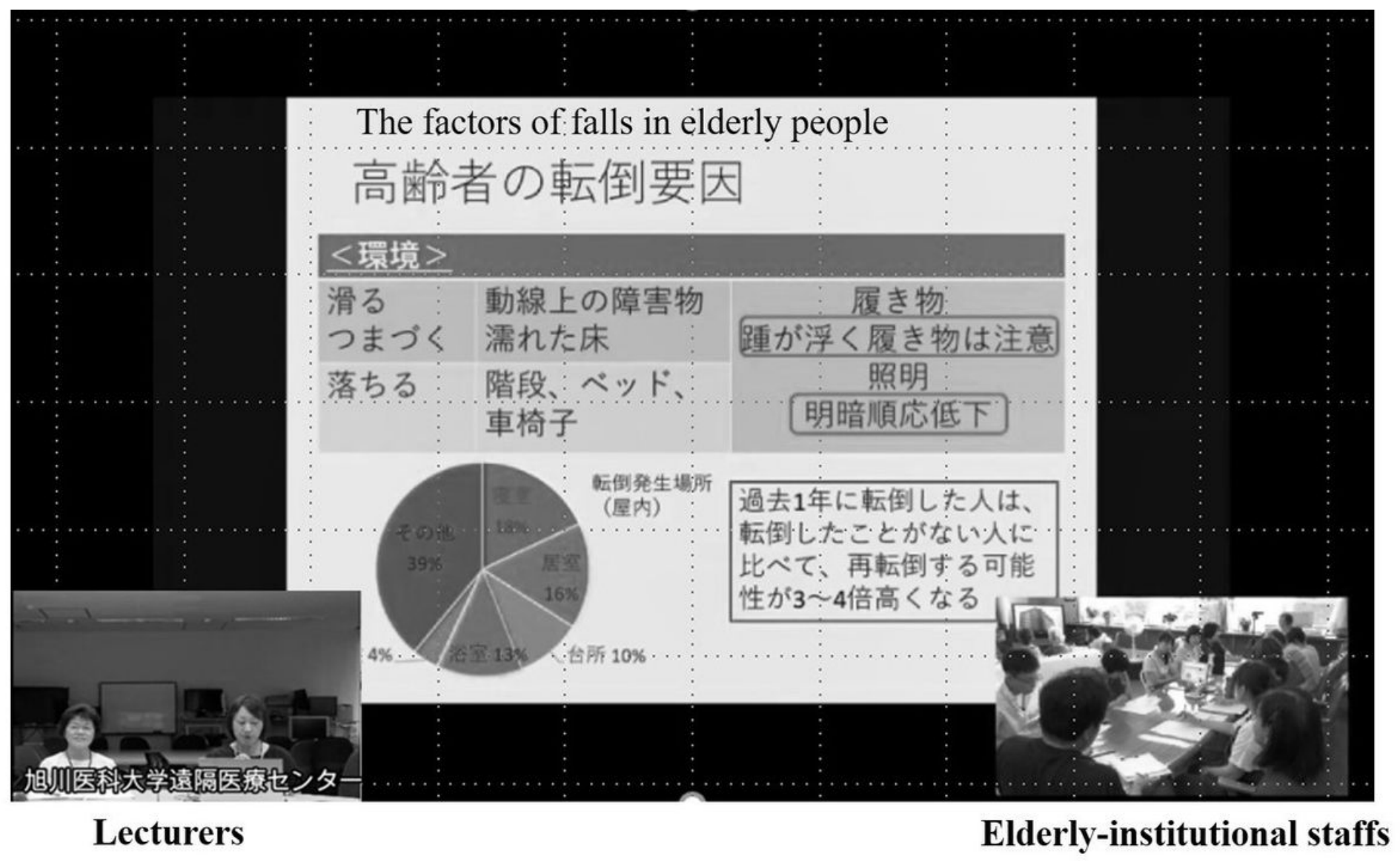

Figure 2

Education for caregivers Lectures were given to staff members of elderly institutions using a telemedicine system and bidirectional discussions were held. 
A

$$
\begin{aligned}
& \text { Date } \\
& \hline \text { Institution } \\
& \hline \text { Name } \\
& \hline
\end{aligned}
$$

Past history

\begin{tabular}{|c|l|c|c|}
\hline \multicolumn{2}{|c|}{ Question } & \multicolumn{2}{|c|}{ answer } \\
\hline 1 & Has your doctor ever said that you have a heart condition? & Yes & No \\
\hline 2 & Do you feel pain in your ehest when you perform physical activity? & Yes & No \\
\hline 3 & $\begin{array}{l}\text { Have you had chest pain when you were not performing any } \\
\text { physical activity? }\end{array}$ & Yes & No \\
\hline 4 & $\begin{array}{l}\text { Do you lose your balance because of dizziness or do you ever lose } \\
\text { consciousness? }\end{array}$ & Yes & No \\
\hline 5 & Is there anyone who died suddenly with unknown cause? & Yes & No \\
\hline 6 & Has your doctor ever said that you have a leg problem or backpain? & Yes & No \\
\hline 7 & Has your doctor diagnosed that you have articular rheumatism? & Yes & No \\
\hline 8 & Have you had a surgery for artificial joint replacement? & Yes & No \\
\hline 9 & Do you have a knee problem in your physical activity? & Yes & No \\
\hline 10 & Do you have a backpain in your physical activity? & Yes & No \\
\hline & & & \\
\hline
\end{tabular}

B

\section{Fall screening sheet}

The indications for fall prevention telerehabilitation

1) The participants can walk with/without walking aids.

2) The participants can move their limbs according to the direction.

Institution:

Name:

Age:

Gender: male - female

Nursing care necessity degree: $1 \cdot 2 \cdot 3 \cdot 4 \cdot 5$

Present illness:

Past history of falls: Yes . No

Usage of mobilization sensor: Yes · No

On going rehabilitation: Yes $\cdot$ No

Past history of stroke:
$\mathrm{C}$

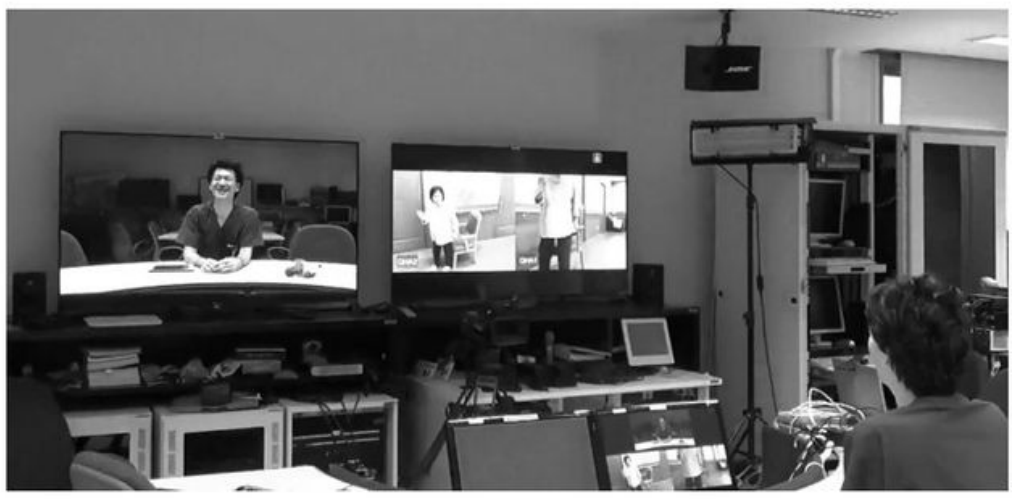

E

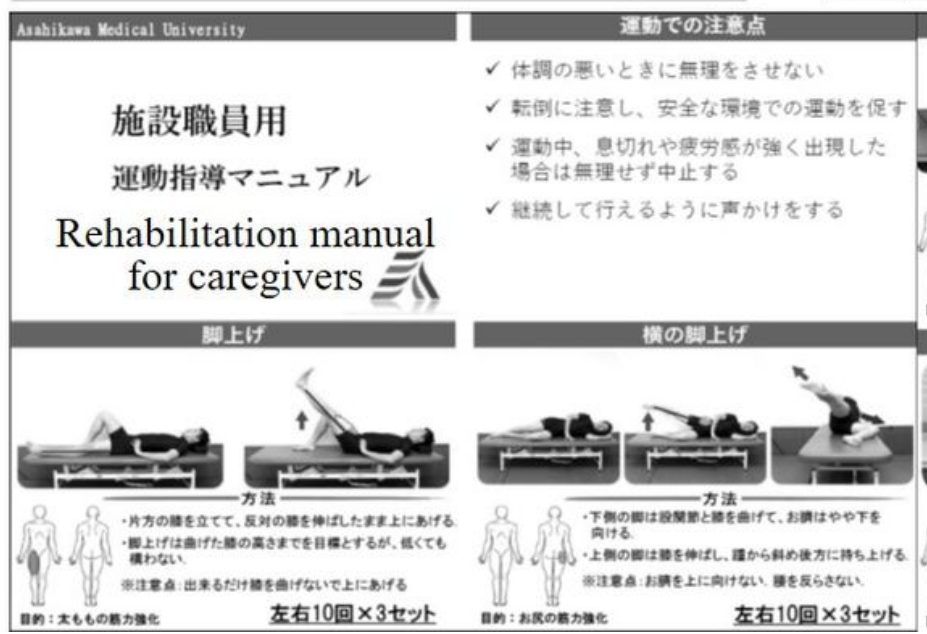

$\mathrm{D}$
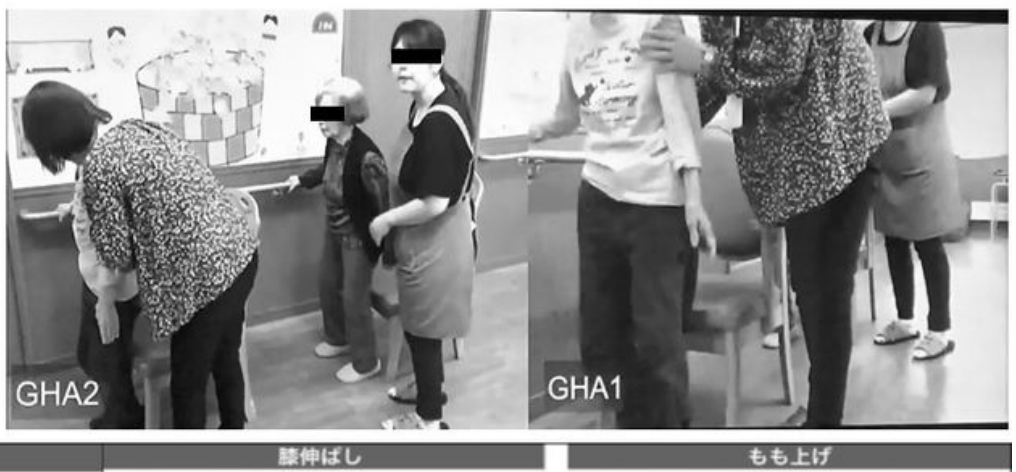

もも上b
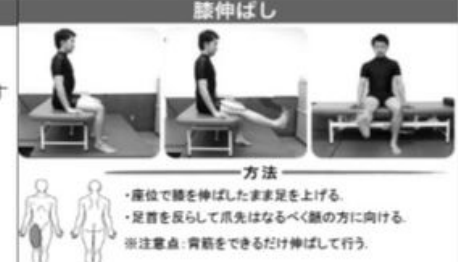

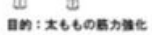

左右10回 $\times 3$ セット
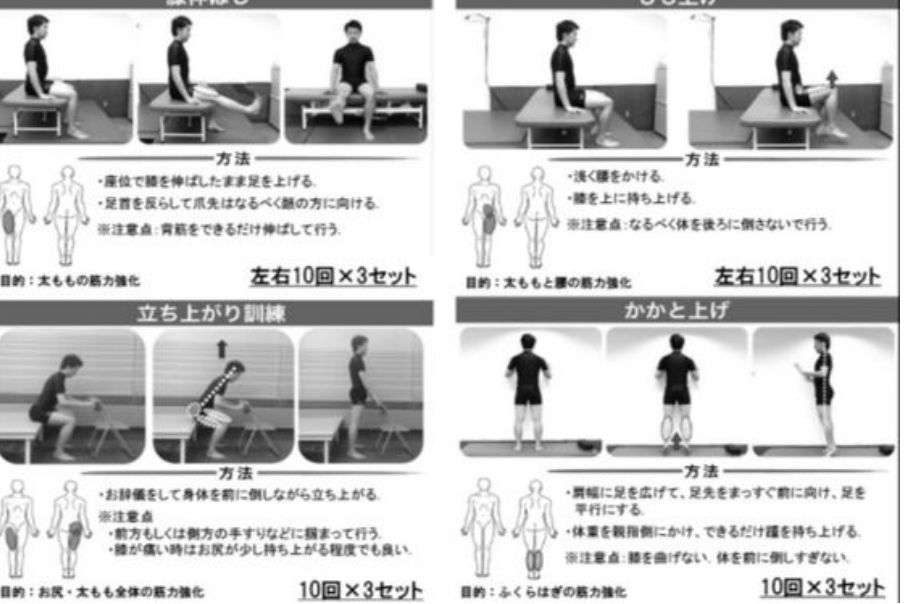

\section{Figure 3}

Questionnaires, telerehabilitation and the rehabilitation manual Questionnaires on the participants' health status (A) and a fall screening sheet (B). MDs, PTs and nurses in Asahikawa Medical University could decide the indications for telerehabilitation based on these questionnaires. Actual scenes of telerehabilitation (C, D) and the rehabilitation manual (E). A PT asked participants about their health condition and directed exercise via the telemedicine system. MDs and PTs proposed a personalized rehabilitation menu based on the rehabilitation manual. 


\section{Pre-post test \\ (score; 0-20)}

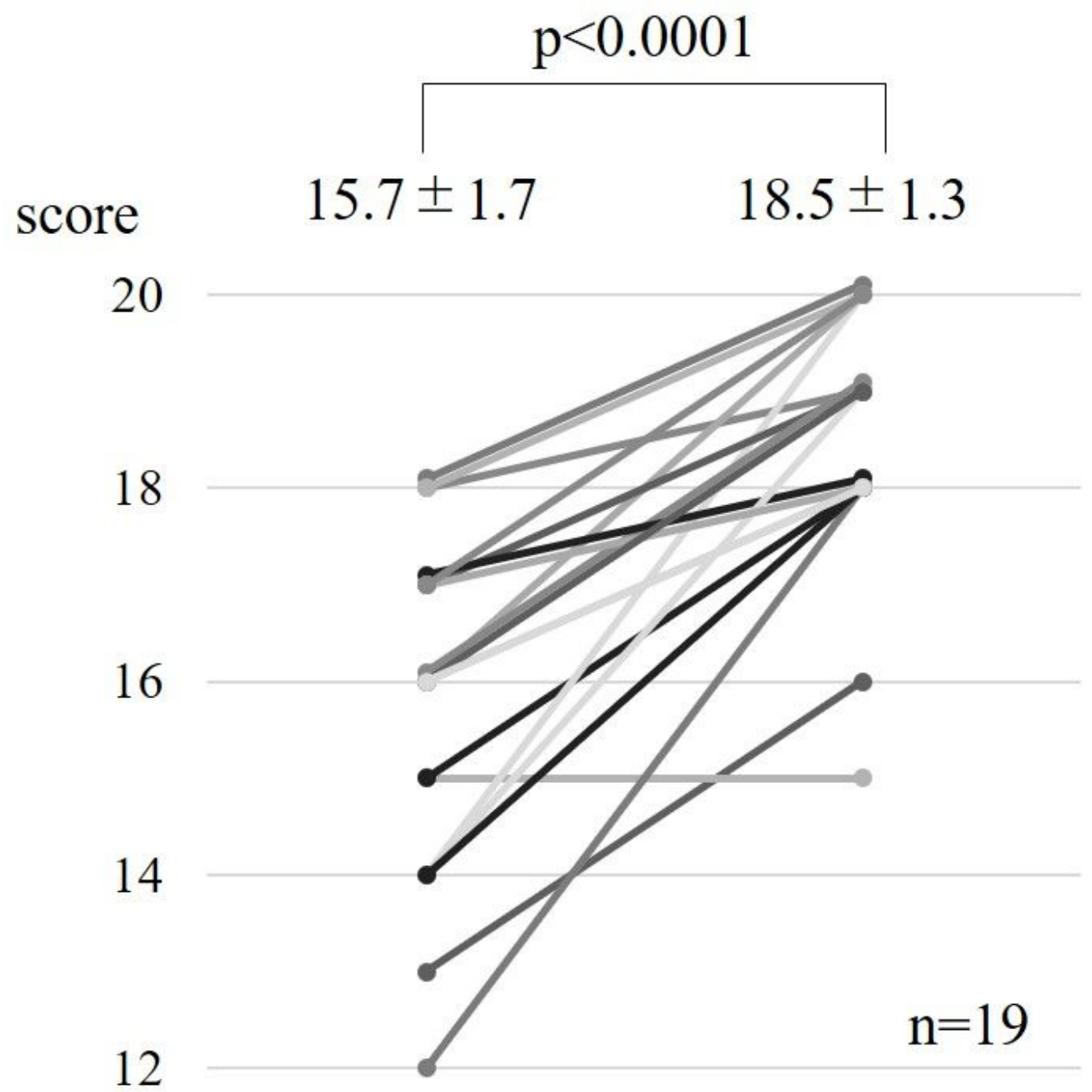

10

\section{Pre-test Post-test}

Figure 4

The efficacy of nursing staff education The lecture significantly improved the total test scores of the nursing staff. 

A. Berg Balance Scale (BBS)
B. Timed Up \& Go test (TUG test)

(Score; 0-56)
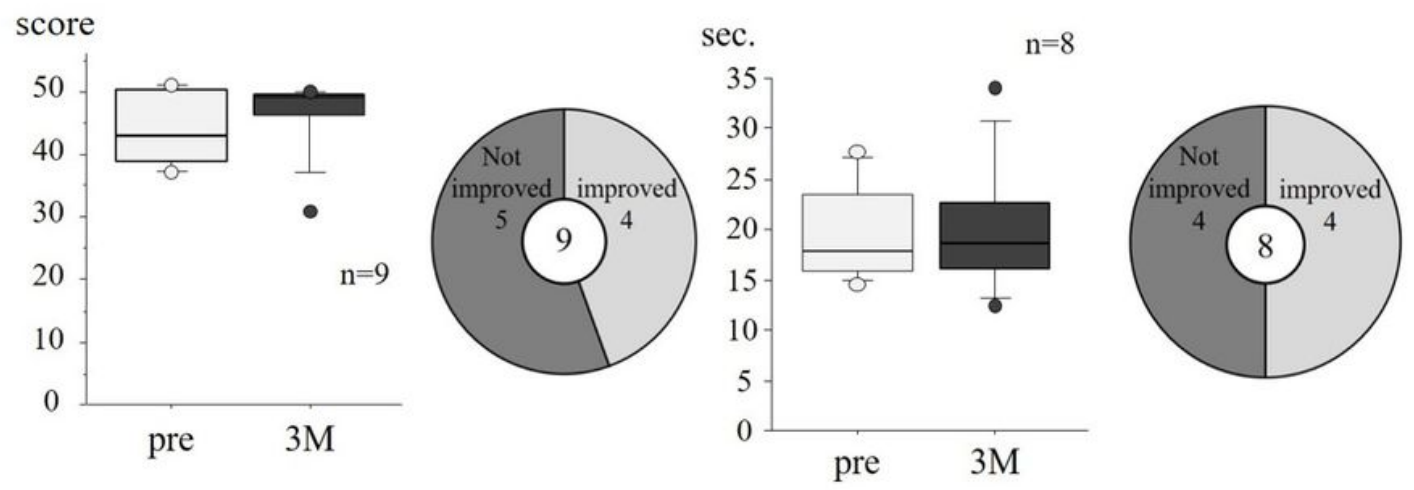

C. Hand-held dynamometer (HHD) (knee extensor muscle strength)

D. Mini-Mental State Examination (MMSE)

$\mathrm{rt}$

lt

(Score; 0-30)

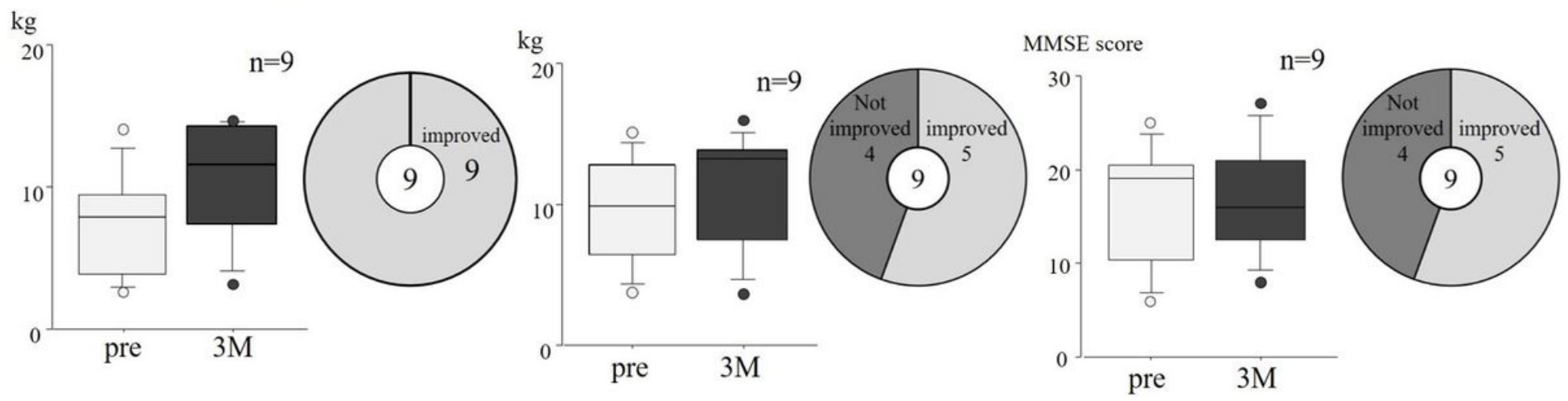

Figure 5

The changes in quantitative physical scales The results of the Berg Balance Scale (BBS) (A), Timed Up \& Go test (TUG test) (B), Hand-held dynamometer (HHD) (C) and Mini-Mental State Examination (MMSE) (D) values are shown. Approximately half of the participants showed improved BBS, TUG, or MMSE values after telerehabilitation. All participants showed improved right lower limb strength. 